childhood. Forty-four per cent of the cases had history of severe eczema occurring early in childhood, probably corresponding to the infective dermatitis associated to the HTLV-I. Myelopathy associated with HTLV-I (HAM) was present in $13.5 \%$ of cases. According to the classification of Shimoyama et al (1991) 22 cases were classified as lymphoma (29.7\%), 21 as smoldering (28.4\%), 19 as acute (25.7\%), and eight as chronic (10.8\%). Four cases (5.4\%) were classified as a new subtype, which has been previously proposed by us: the primary cutaneous tumoral form that is characterized by the absence of lymphadenomegaly, lymphocytosis, leukemia, hypercalcemia and involvement of internal organs in the presence of normal or slightly elevated LDH levels. I suggest that this new subtype should be included in Shimoyama's classification, since although the cases have only skin lesions they are tumoral and has a worse prognosis than the smoldering type (Table 1). So we would have five subtypes: 1. smoldering with cutaneous non-tumoral lesions; 2. primary cutaneous tumoral; 3. chronic; 4. acute; 5 . lymphoma (tumors in lymph nodes and/or in internal organs). In forty-one cases (55.4\%) the lesions began in the skin and were considered as a primary cutaneous form. In table 1, are referred the duration of disease, survival and frequency of deaths in each clinical type. Seventy seven per cent of the patients are dead and one has been lostto-follow-up.

Histologically, a diagnosis of non-specific peripheral T-cell lymphoma was made in 52 cases (70.2\%), while 16 cases (21.6\%) were diagnosed as mycosis fungoides and 6 cases (8.2\%) as anaplastic large cell lymphoma. In 18 of the 74 cases studied clonal integration by southern blot or inverse PCR was evaluated with positive results. Molecular studies are in course in the other cases.

Some aspects observed in these ATL cases were not frequently reported in the literature: 1) earlier appearance, including the occurrence in two adolescents and one child; 2) occurrence of a new clinical subtype, the primary cutaneous tumoral form; 3) presence of other morphological types of lymphoma besides the nonspecific peripheral T-cell lymphoma (mycosis fungoides and anaplastic large cell lymphoma), 4) higher association with HAM(13.5\%); and 5) frequent history of severe eczema in childhood.

Table 1. Duration of disease, survival and frequency of deaths among 74 cases of ATL

\begin{tabular}{lcccc}
\hline Clinical types & $\mathbf{N}^{\mathbf{0}}$ cases (\%) & $\begin{array}{c}\text { Duration of } \\
\text { disease (years) }\end{array}$ & Survival (years) & Deaths (\%) \\
\hline Lymphoma & $22(29.7)$ & 0.3 & 1.9 & 95.5 \\
Smoldering & $21(28.4)$ & 3.6 & 3.9 & 42.0 \\
Acute & $19(25.7)$ & 0.5 & 0.6 & 94.7 \\
Chronic & $8(10.8)$ & 2.1 & 2.3 & $87.5 \%$ \\
Primary cutaneous tumoral & $4(5,4)$ & 0.9 & 1.8 & 75.0 \\
\hline
\end{tabular}

This study was supported by grants from the Conselho Nacional de Pesquisa (CNPq) and Fundação de Apoio à Pesquisa na Bahia (FAPESB).

\title{
HIV Associated Lymphomas: Problems with the Histopathologic Classification
} Helenemarie Schaer Barbosa

The incidence of B-cells lymphoma in HIV infected individuals is increased up to 100 times. These lymphomas are usually aggressive, of high grade, disseminated and frequently extranodal. The most frequent histological types are diffuse large B cell and Burkitt's lymphomas. Recently, two new histological types were described almost exclusively in AIDS patients: Primary Effusion Lymphoma (PEL) and Plasmablastic Lymphoma. Typically Primary Effusion Lymphomas present as a cavitary effusion containing malignant neoplastic cells. Occasionally they present 
solid tumors simultaneously with the effusion or during the course of disease. Almost $100 \%$ of these cases are $\mathrm{HIV}+$, the tumor cells are HHV8+, and more than 2/3 of the cases are EBV+. The malignant cells resemble immunoblasts and sometimes they show plasmacytoid differentiation. Half of the patients express CD45R(LCA) and 20\% express CD20 (pan-B). They can also express CD38, a marker of plamocytes. The present discussion about PEL is the finding of several solid tumors with similar characteristics, HHV8+, but without effusion during the whole course of disease. Knowles, Chadburn and Cesarman's group support the idea that these cases are inside the PEL spectrum and that they should be classified as extra-cavitary variant of the PEL. On the other hand, the plasmablastic lymphoma, EBV+ and HHV8- described originally from the oral mucosa, which is a solid tumor with no effusion, has been found in other sites like nasal and paranasal sinuses, testicle, bone and anorectal regions and a few are HHV8+. Morphologically, the plasmablastic lymphoma is very similar to PEL. The malignant cells have plasmablastic differentiation (an immunoblast with plasmacytoid differentiation), in other terms they are similar to immunoblasts, large cells with vesicular nucleus and a prominent nucleolus that usually do not express normal B cell markers such as CD20, but express plasmacytoid differentiation markers such as CD38. Even the immunocytochemical profile is similar to PEL. The situation is complicated by a recent publication of HIV patients with multiple mieloma developing extramedular solid tumors with plasmablasts of similar morphology to PEL and plasmablastic lymphoma. In conclusion, patients with AIDS can present lymphomas with plasmablastic differentiation, associated or not to HHV8, generally EBV+ that sometimes present with cavitary effusion, some other times are solid tumors or even mixed. If they are part of the spectrum of the same lymphoma or distinct entities, it is not quite clear. With the present knowledge, I believe that it is too soon to classify these lymphomas, before more is known about them. To designate a solid tumor as a solid variant of effusion primary lymphoma, looks to me farfetched and does not contribute to clarify the disease. The role of HHV8 and EBV in the genesis of these lymphomas with plasmablastic differentiation need further studies.

\section{Aggressive Non-Hodgkin's Lymphoma - Therapeutic Considerations of Pre Rituximab Era Carmino Antônio de Souza}

Lymphomas have been known as one of the most responsive malignancies to chemotherapy. They are frequently subdivided on Hodgkin's Disease (HD) and Non-Hodgkin's Lymphoma (NHL). Lymphomas are heterogeneous clonal malignancies that have been classified in different ways. For the REAL classification, each disease is defined as a different entity based on the morphology integration, immunephenotypic and genetics aspects, clinical behavior and the original cell. Most patients with localized disease, stage I and II (Ann Arbor Classification), cure with conventional doses of chemotherapy (CTX) or CTX + radiotherapy (RTX) - 75\% of 5 years disease-freesurvival (DFS). Those with advanced disease (stage III and IV) have less favorable results, the complete remission rate (RR) is nearly $50 \%$, but only $2 / 3$ of the patients will be free of disease. The long term DFS for these patients is around 40 to $50 \%$ with polichemotherapy. Regimens with antracyclines, specifically CHOP (cyclophosphamide (CY), doxorubicin, vincristine, e prednisone), have been the main approach for aggressive lymphomas since 1970.70 to $80 \%$ of the patients will respond to CHOP. The long term cure rate with CHOP is around 35\% and there are no evidences that second and third generation regimens are more efficient. FISHER, in 1993 did not find difference in the survival and response rates comparing CHOP, m-BACOD, Promace-Cytabom and MACOP$\mathrm{B}$, although the CHOP protocol was cheapest and less toxic. GORDON, SERTOLI e COOPER also did not show that regimens like m-BACOD, MACOP-B, e ProMACE-MOPP, have better results than CHOP on the treatment of intermediate and high grade lymphomas. It is generally accepted that patients with intermediate and high grade lymphomas that relapse after initial therapy have a reserved prognostic and the additional 